\title{
Stimulated Raman Scattering in Quantum Dots and Nanocomposite Silicon Based Materials
}

\author{
M. A. Ferrara*, I. Rendina and L. Sirleto \\ National Research Council-Institute for Microelectronics and Microsystems, Napoli,
} Italy

\section{Introduction}

Stimulated Raman Scattering (SRS) is one of the first discovered nonlinear optical effects: a pump laser beam enters a nonlinear medium and spontaneous generation and amplification lead to a beam at a frequency different from the pump. SRS is dependent on the pump intensity and on a gain coefficient $g$, which depends on material scattering efficiency: the larger the spontaneous scattering efficiency of materials is, the higher the Raman gain for a given intensity is obtained. As a general rule, there is a trade-off between gain and bandwidth in all laser gain materials: line-width is bought at the expense of peak gain. Of course, this is true for bulk solids, but the question is what happens at nanoscale?

Nonlinear optics at nanoscale is a recent fascinating research field. Stimulated Raman scattering in electrons-confined and photons-confined materials is of great importance from both fundamental and applicative point of view. Concerning the fundamental one, there have been a number of investigations both experimental and theoretical, but the question is still "open", while from an applicative point of view, there are some important prospective, for example to realize micro/nano source, with improved performances.

In this chapter, experimental investigations of stimulated Raman scattering in silicon quantum dots and in silicon nanocomposite are reported. Two Raman amplifiers are realized and amplifications due to stimulated Raman scattering are measured. For both of them, a significant enhancement of Raman gain and a significant reduction in threshold power are demonstrated. Our findings indicate that nanostructured materials show great promise for Si-based Raman lasers.

\section{Basis on Stimulated Raman Scattering and Raman amplifier}

SRS belongs to a class of nonlinear optical processes that can be called quasi-resonant. Although none of the fields is in resonance with the vibrations in the lattice of the medium (optical phonons), the difference between the pump and generated beam equals the transition frequency. SRS is used in tunable laser development, high energy pulse compression, etc.

SRS can be obtained by irradiating a solid with two simultaneous light sources: a light wave at frequency $\omega_{L}$ (the pump laser wave) and a light wave at frequency $\omega_{S}=\omega_{L}-\omega_{v}$ (the

${ }^{*}$ Corresponding Author 
Stokes Raman wave), where $\hbar \omega_{v}$ corresponds to a vibrational energy (Fig. 1). The pump causes the molecular vibration and thereby impress frequency sidebands (Stokes and antiStokes). From the other side, Stokes wave at frequency $\omega_{S}$ can beat the laser to produce a modulation of the total intensity that coherently excites the molecular oscillation at the frequency $\omega_{v}=\omega_{L}-\omega_{S}$. These two process due to pump and Stokes waves, reinforce one another in the sense that the pump effect leads to a stronger Stokes wave, which in turn leads to a stronger molecular vibration (Boyd, 2003).

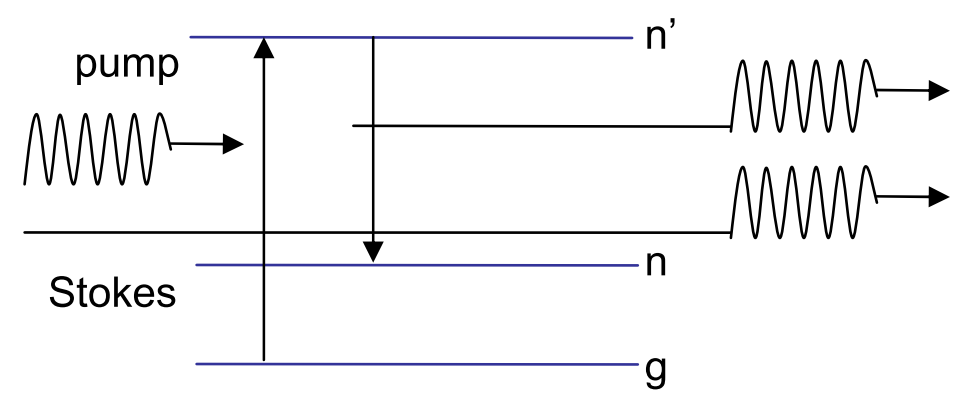

Fig. 1. Stimulated Raman Scattering.

In the steady-state (no pump depletion) regime of SRS, the intensity of the output Stokes radiation is expressed by (Shen \& Bloembergen , 1965)

$$
I_{S}(L)=I_{S}(0) \cdot \exp \left(I_{P}(0) g L\right)
$$

where $I_{S 0}$ is the intensity of the input Stokes radiation (Stokes seed), $I_{S}$ is the intensity of the output Stokes radiation, $I_{P}$ is the intensity of the pump radiation, $g$ is the Raman gain coefficient, and $L$ is the effective length. Assuming no losses at the Stokes frequency, the value of the gain coefficient $g$ can be obtained by fitting Eq. (1), which is readily transformed into

$$
S R S=10 \cdot \log _{10}\left(\frac{I_{S}(L)}{I_{S}(0)}\right)=4.34 \cdot g L I_{P}(0)
$$

where $I_{P}=P / A$ with $P$ as the power incident onto the sample and $A$ as the effective area of pump beam. Since the sample transparent to the incident light, $L$ is taken to be equal to the thickness of the sample along the path of the incident light.

Raman lasing can be achieved by using the Stimulated Raman Scattering phenomenon, which permits, in principle, the amplification in a wide interval of wavelengths, from the ultraviolet to the infrared.

Fused silica has been, for the past century, the key material used for long and short haul transmission of optical signals, because of its good optical properties and attractive figure of merit (i.e. trade-off between Raman gain and losses). A breakthrough in fiber optics communications was achieved with the reduction of the water absorption peak at $1400 \mathrm{~nm}$, which opened up the available communication range to span from 1270 to $1650 \mathrm{~nm}$, corresponding to about $50 \mathrm{THz}$ bandwidth (Rivero et al., 2004). This dramatic increase in bandwidth rules out the use of existing Er doped fiber amplifiers, leaving Raman gain as the 
main mechanism for future amplification needs. However, the main disadvantage of the current silica fiber amplifiers is the limited usable bandwidth for Raman amplification (5 $\mathrm{THz}$, approx. $150 \mathrm{~cm}^{-1}$ ).

On the other hand, in the past few years several strategies have been developed to engineer efficient light sources and amplifiers in silicon-based materials (Pavesi \& Lockwood, 2004; Soref, 2006), with the aim to demonstrate a convenient path to monolithic integration of optical and electronic devices within the mainstream Si technology. In particular, Raman amplification is an interesting approach for optical amplification, because it is only restricted by the pump wavelength and Raman active modes of the gain medium (Islam, 2002; Mori et al., 2003). Light amplification by stimulated Raman scattering in silicon waveguides has been recently demonstrated, despite intrinsic limitations related to the nature of the bulk Si materials have been pointed out (Jalali et al., 2006; Dekker et al., 2007).

Raman scattering in bulk silicon was studied as early as 1965 (Russell, 1965). Using a helium-neon laser with an output wavelength of $0.6328 \mu \mathrm{m}$, backward Raman scattering from silicon was measured, and it was found that the Raman scattering efficiency in silicon was 35 times larger than that for diamond (Russell, 1965). In 1970 (Ralston \& Chang, 1970), more detailed Raman experiment using a YAG:Nd laser having a wavelength of $1.064 \mu \mathrm{m}$ was performed. Both spontaneous and stimulated Raman scattering efficiency was characterized experimentally (Ralston \& Chang, 1970). The observed Raman frequency downward shift of $15.6 \mathrm{THz}$ corresponds to optical phonon energy of silicon at the center of the Brillouin zone (Hart et al., 1970; Temple \& Hathaway, 1973). The first-order resonance, which is of primary importance here, has a full-width at half-maximum of $105 \mathrm{GHz}$ (Temple \& Hathaway, 1973). This imposes a maximum information bandwidth of approximately 105 $\mathrm{GHz}$ that can be amplified. The Raman linewidth becomes broader when a broadband pump is used. These experiments has the merit to prove that silicon has a relatively strong Raman scattering efficiency (four orders of magnitude higher than that for silica) (Agrawal, 1995; Claps et al., 2002; Claps et al., 2003).

\subsection{SRS in silicon waveguide}

SRS has been exploited in optical fibers to create amplifiers and lasers. However, several kilometers of fiber is typically required to create a useful device, suggesting that the approach is not applicable in integrated devices. Often overlooked was the fact that the gain coefficient for SRS in silicon is approximately $10^{3-10^{4}}$ times higher than that in silica fiber. Additionally, owing to the large refractive index, silicon waveguides can confine the optical field to an area that is approximately 100-1000 times smaller than the modal area in a standard single-mode optical fiber, resulting in proportionally higher Raman gain (Claps et al., 2002). When combined, these facts make it possible to observe SRS over the interaction lengths encountered on a chip (Claps et al., 2002; Claps et al., 2003; OSA Press Room Editorial).

As silicon is transparent in 1.3-1.6 $\mu \mathrm{m}$ (optical communication band), Raman scattering in silicon waveguides in such a wavelength range has attracted a great deal of interest in the past couple of years (Claps et al., 2002; Rong at al., 2005).

The use of SRS in silicon waveguides was proposed in 2002 as a means to realize silicon amplifiers and lasers (Claps et al., 2002; OSA Press Room Editorial). This was followed by 
demonstration of stimulated emission (Claps et al., No. 15, 2003) and Raman wavelength conversion (Claps et al., No. 22, 2003) in silicon waveguides in 2003. Shortly afterward, the approach led to the demonstration of the first silicon laser in 2004: a device that operated in the pulsed mode (Boyraz \& Jalali, 2004; Nature News Editorial) followed by demonstration of continuous-wave (CW) lasing in 2005 (Rong et al., No. 7027, 2005).

Pulsed lasing has been achieved using a ring cavity formed by an 8-m-long optical fiber and silicon as the gain medium via SRS (Boyraz \& Jalali, 2004). The slope efficiency, which is described by the ratio of the output peak power and the input peak pump power, obtained was $8.5 \%$.

An all-silicon Raman CW laser has been demonstrated using centimeter-size silicon waveguide (Rong et al., No. 7023, 2005; Rong et al., No. 7027, 2005). Recently, the waveguide was replaced by a ring cavity witch have a total length of $3 \mathrm{~cm}$ and a bend radius of $400 \mu \mathrm{m}$ (Rong et al., 2006). A slope efficiency of $\sim 10 \%$ was obtained.

\subsection{Problems in silicon waveguide Raman amplifiers}

In two-photon absorption (TPA) process an electron absorbs two photons from the laser (Fig. 2) at approximately the same time (or within less than a nanosecond) and achieves an excited state that corresponds to the sum of the energy of the incident photons. This is a nonlinear process, occurring with significant rates only at high optical intensities, because the two-photon absorption coefficient is proportional to the optical intensity of pump laser. TPA can be eliminated entirely choosing a material for which the lowest-lying excited state lies more than $2 \hbar \omega$ above the ground state (Boyd, 2003).

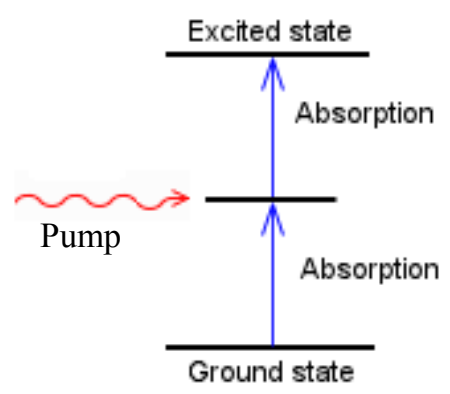

Fig. 2. Two Photon Absorption.

As a multiphoton process, TPA is a competitive effect of stimulated Raman scattering. In particular the two-photon absorption (TPA) reduces the efficiency of SRS phenomena.

The main challenge in silicon Raman laser is the loss caused by the free carriers that are generated via TPA (Liang \& Tsang, 2004; Claps et al., 2004). By determining the steady-state density of generated carriers, and hence the magnitude of the pump-induced loss, the recombination lifetime is the central parameter in Raman as well as other semiconductor nonlinear optical devices. Due to the presence of interface states at the boundary between the top silicon and the buried oxide layer, the recombination lifetime in SOI is much shorter than that in a bulk silicon sample with comparable doping concentration. This effect 
depends on the method used for preparation of the SOI wafer and the film thickness, with measured and expected values ranging between 10 and 200 ns (Mendicino, 1998; Freeouf \& Liu, 1995). In SOI waveguides, the lifetime is further reduced to a few nanoseconds, or even below in the case of submicrometer waveguides, due to the recombination at the etched waveguide facets and, in the case of rib waveguides, due to diffusion into the slab regions (Dimitropoulos et al., Vol. 86, 2005; Espinola et al., 2004). By introducing midgap states through high-energy irradiation and gold or platinum doping, the lifetime can be further reduced. The carrier density can also be reduced by using a reverse-bias p-n junction to sweep the carriers out (Liang \& Tsang, 2004; Claps et al., 2004), and CW gain using this approach has been reported (Liu et al., 2004; Fathpour et al., 2006). However, free-carrier screening of the junction electric field, a phenomenon reminiscent of high power saturation in photodetectors, limits the usefulness of this technique to modest pump intensities (Dimitropoulos et al., Vol. 87, 2005). Furthermore, the diode results in electrical power being dissipated on the chip.

Being optically pumped, it is unlikely that the Raman laser will play a role in optical interconnects; on the other hand, by compensating for coupling and propagation losses, a Raman amplifier can have an impact. However, the device length will have to be drastically lower than the centimeter-length device demonstrated so far. At an intensity of $100 \mathrm{MW} / \mathrm{cm}^{2}$ (100 $\mathrm{mW}$ pump coupled into a $0.1 \mu \mathrm{m}^{2}$ waveguide) and with silicon's Raman gain coefficient of $\sim 20 \mathrm{~cm} / \mathrm{GW}$, the gain will be $\sim 1 \mathrm{~dB} / \mathrm{mm}$. Such a modest gain per unit length creates a challenge for miniaturization of Raman amplifiers, a prerequisite for their integration with silicon VLSI-type circuits. The long waveguide length will not be an issue if the device is used as a stand-alone discrete amplifier (similar to the role played by the EDWA).

\subsection{Silicon nanostructures}

Taking into account intrinsic limitations related to the nature of the bulk Si materials, that is the narrow-band $(105 \mathrm{GHz})$ of stimulated Raman gain, the small Raman effect and the competing nonlinear effect of TPA, which reduces the efficiency of SRS, the investigation of new materials possessing both large Raman gain coefficients and broader spectral bandwidth than fused silica and/or silicon is becoming mandatory in order to satisfy the increasing telecommunications demands.

Nanostructured silicon has generated large interest in the past decades as a promising key material to establish a Si-based photonics. An accurate knowledge of both linear and nonlinear optical properties of these structures is crucial for the conception and design of highly efficient photonic structures and for the control of their performance (Pavesi \& Lockwood, 2004).

Strong enhancement $\left(\sim 10^{3}\right)$ of the spontaneous Raman scattering was reported from individual silicon nanowires and nanocones as compared with bulk Si (Cao et al., 2006). The observed enhancement was diameter, excitation wavelength, and incident polarization state dependent. The observed increase in Raman-scattering intensity with decreasing diameter in this system was explained in terms of structural resonances in the local field similar to Mie scattering from dielectric spheres.

We note that SRS from spherical droplets and microspheres, with diameters $5 \div 20 \mu \mathrm{m}$, has been observed using both pulsed and continuous wave probe beams (Spillane et al., 2002). 
Except for report of SRS from individual single walled carbon nanotubes (Zhang et al., 2006), and the observation of SRS from semiconductor nanowires (Jian Wu et al., 2009), we find no other evidence for this important nonlinear optic effect in nanostructured materials.

\section{Broadening and tuning of Spontaneous Raman Scattering in silicon nanocrystals}

In this paragraph, some advantages of Raman approach in silicon nanocrystals with respect to silicon are theoretically and experimentally demonstrated. In order to provide theoretical basis for these results, phonon confinement model is briefly introduced. After that, according to this model, we discuss two significant improvements of this approach: the broadening of Raman scattering and the tuning of Stokes shift in Si-nc with respect to silicon.

When the size of the particle reduce to the order of $\mathrm{nm}$, the wave function of optical phonons will non longer be a plane wave. The localization of wave function leads a relaxation in the selection rule of wave vector conservation. Not only the phonons with zero wave vector $\mathrm{q}=0$, but also those with $\mathrm{q}>0$ take part in the Raman scattering process, resulting in the red shift of the peak position and the broadening of the peak width. A quantitative model, developed by Campbell and Fauchet, calculates that the peak position mainly depends on the number of atoms included in a cluster, while the width of spectra depends on the shape of crystallites (Ritcher et al, 1981; Campbell \& Fauchet, 1986).

Silicon nanocrystals sample can be modelled as an assembly of quantum dots and the phonon confinement is three dimensional. The weight factor of the phonon wave function is chosen to be a Gaussian function as follows:

$$
W(r, L)=\exp \left(-\frac{8 \pi^{2} r^{2}}{L^{2}}\right)
$$

where $\mathrm{L}$ is the average size of dots. The Square of the Fourier transform is given by:

$$
|C(q)|^{2}=\exp \left(-\frac{q^{2} L^{2}}{16 \pi^{2}}\right)
$$

The first-order Raman spectrum $\mathrm{I}(\omega)$ is thus given by:

$$
I(\omega) \cong \int \exp \left(-\frac{q^{2} L^{2}}{16 \pi^{2}}\right) \frac{d^{3} q}{[\omega-\omega(q)]^{2}+\left(\frac{\Gamma}{2}\right)^{2}}
$$

where $\mathrm{q}$ is expressed in units of $\frac{2 \pi}{a}$ and $\mathrm{a}=0.54 \mathrm{~nm}$ is the lattice constant of silicon, $\Gamma$ is the natural line width for c-Si at room temperature $\left(3.5 \mathrm{~cm}^{-1}\right)$ and $\omega(\mathrm{q})$ is the dispersion relation for optical phonons in c-Si which can be taken according to:

$$
\omega(q)=\omega_{0}-120\left(\frac{q}{q_{0}}\right)^{2}
$$


where $\omega_{0}=520 \mathrm{~cm}^{-1}$ and $q_{0}=2 \pi / a_{0}$.

It was proved that the phonon confinement model is suitable to fit experimental results (Sirleto et al., 2006).

Experimental measurements proving broadening and tuning of Raman spectra have been also performed (Ferrara et al., 2008). Unpolarised Raman spectra have been detected at room temperature in backscattering geometry using a Jobin Yvon Ramanor U-1000 double monochromator, equipped with a microscope Olympus BX40 for micro-Raman sampling and an electrically cooled Hamamatsu R943-02 photomultiplier for photon-counting detection. The excitation source was a Coherent Innova 70 argon ion laser, operating at 514.5 $\mathrm{nm}$ wavelength. In order to prevent laser-annealing effects, the average laser power was about $2 \mathrm{~mW}$ at the sample surface. Using a 50X objective having long focal distance, the laser beam was focused to a diameter of few microns. Its position on the sample surface was monitored with a video camera. All components of the micro-Raman spectrometer were fixed on a vibration damped optical table.

In Fig.3, the Raman spectra of samples of Si-nc having different size are shown. As expected on the basis of the phonon confinement model (Campbell \& Fauchet, 1986; Ritcher at al, 1981), the red shift and the asymmetry of the PS Raman peak increase with decreasing size of Si-nc.

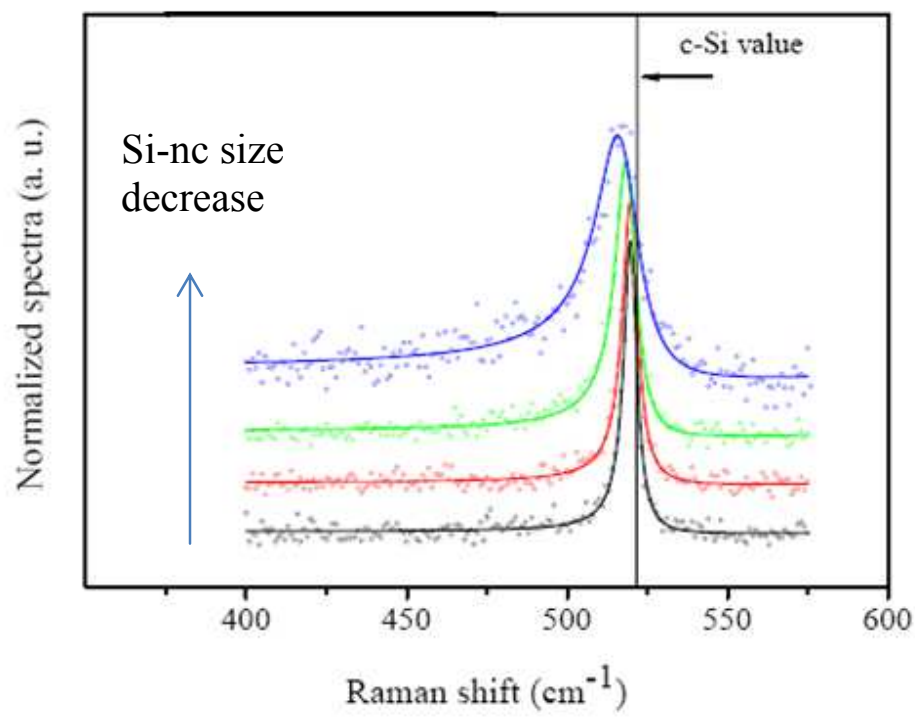

Fig. 3. Raman spectra (circles) of Si-nc samples with different size of nanocrystals.

According to the phonon confinement model, in Fig.4 the peak width of spontaneous Raman emission as a function of nanocrystal size is reported. It is possible to note that the peak width has an inverse dependence on crystal size. Furthermore, considering silicon nanocrystals having crystal size of $2 \mathrm{~nm}$, a significant broadening could be obtained (bandwidth of about $65 \mathrm{~cm}^{-1}$ ). 
Moreover, according to the same model, in Fig.5 the peak shift of spontaneous Raman emission as a function of nanocrystal size is reported. Also the peak shift has an inverse dependence on nanocrystal size. For nanocrystal of about $2 \mathrm{~nm}$ a peak shift of about $19 \mathrm{~cm}^{-1}$ can be obtained. Because the width of C-band telecommunication is $146 \mathrm{~cm}^{-1}$, taking into account the broadening and the shift of spontaneous Raman emission, we conclude that more than the half of C-band could be cover considering Si-nc, without implementing the multi-pump scheme.

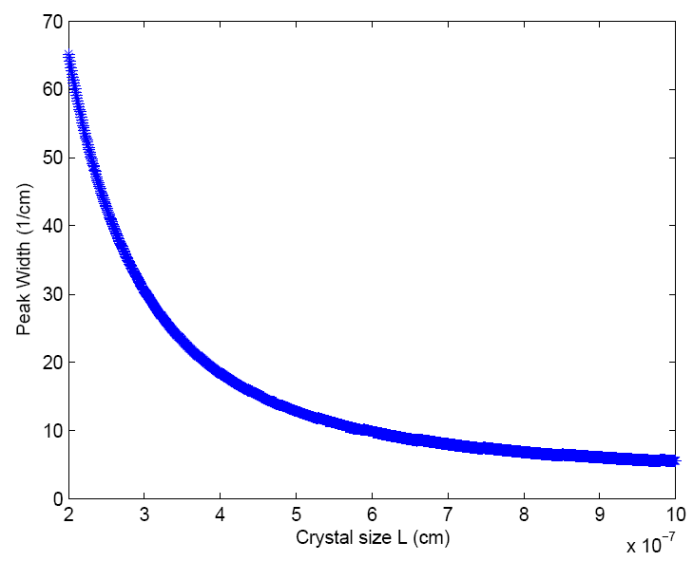

Fig. 4. Calculated relationship between the Raman peak width and the nanocrystal size.

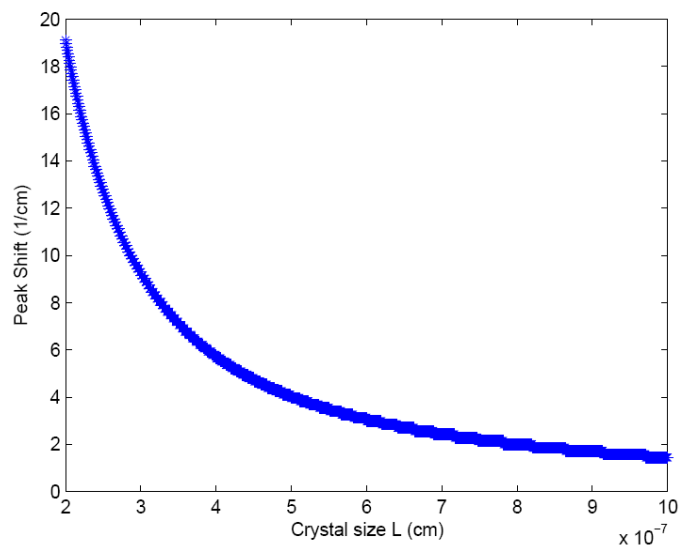

Fig. 5. Calculated relationship between the Raman peak shift and the nanocrystal size.

\section{Stimulated Raman Scattering measurements}

According with the results discussed above, in this section are reported measurements of stimulated Raman scattering in two different sample: silicon nanocrystals embedded in Sirich nitride/silicon superlattice structures (SRN/Si-SLs) and silicon nanocomposities embedded in $\mathrm{SiO}_{2}$. 


\subsection{Experimental set-up}

In Fig. 6, the experimental setup used in order to measure SRS is shown. The pump laser is a CW pump-Raman laser operating at $1427 \mathrm{~nm}$. The probe laser is a tunable external cavity diode laser (1520-1620 nm). The probe beam is split by a Y fiber optic junctions. One of the branches is used in order to monitor probe fluctuations. The other one and the pump laser are combined on a dichroic mirror and subsequently coupled to a long working distance 50X infrared objective in order to be focused onto the sample. The sample is mounted parallel to the path of the incident beam. Estimated coupling losses were about $4 \mathrm{~dB}$. The transmitted signals from the sample are collected by a 20X microscope objective. In order to separate the probe from the pump, a dichroic filter and a longpass filter were used. An optically broadband photodetector (PD) was used to collect the probe signal. The signal from the PD is demodulated by a lock-in amplifier, which is externally referenced to the $180 \mathrm{~Hz}$ chopper. Each data point is averaged 1000 times before being acquired. Additionally, four measured values are averaged for each data point. The accuracy of measurement is $\pm 0.1 \mathrm{~dB}$.

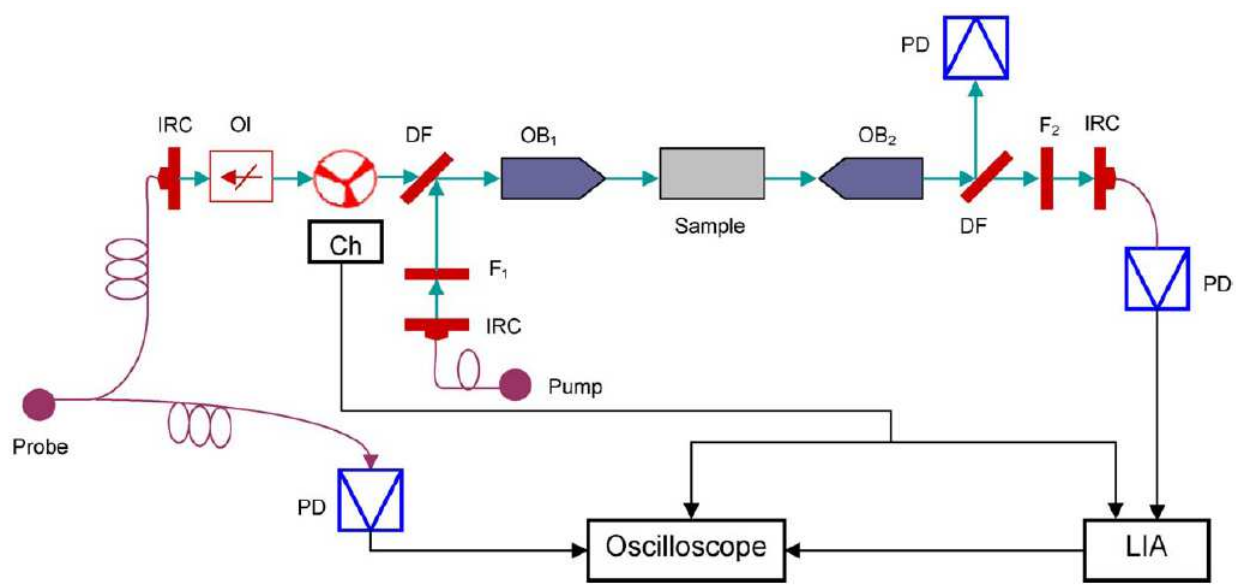

Fig. 6. Experimental setup for SRS measurements: pump-Raman laser; IRC-infra-red collimator; F1-bandpass filter at $1427 \mathrm{~nm}$; probe: ECDLexternal cavity diode laser (tunable); OI-optical insulator; DF-dichroic filter; OB1 (OB2)-microscope objective lens 50X (20X); F2longpass filter at $1500 \mathrm{~nm}$; Ch-Chopper; PD-optically-broadband photodetector; LIA-Lockin amplifier. Black lines represent electrical connections and wiring, green lines represent freespace optical beams, and magenta lines represent optical fiber.

\subsection{Samples preparation and structural characterization}

Raman amplification measurements were performed both on silicon nanocrystals embedded in Si-rich nitride/silicon superlattice structures (SRN/Si-SLs) and on silicon nanocomposities embedded in $\mathrm{SiO}_{2}$.

SRN/Si-SLs samples were fabricated on $\mathrm{Si}$ substrates by radio frequency (RF) magnetron cosputtering from $\mathrm{Si}$, and $\mathrm{Si}_{3} \mathrm{~N}_{4}$ targets. The sputtering of the multilayer structures was performed in a Denton Discovery 18 confocal-target sputtering system, as described elsewhere (Dal Negro et al., 2008). An atomic concentration of $48 \%$ Si was measured in the 
deposited films with energy dispersive $x$-ray analysis (Oxford ISIS). The multilayer structure has been annealed using a rapid thermal annealing furnace in $\mathrm{N}_{2} / \mathrm{H}_{2}$ forming gas (5\% hydrogen) for $10 \mathrm{~min}$ at $800{ }^{\circ} \mathrm{C}$. Thermal annealing resulted in a phase separation process leading to the nucleation of amorphous Si clusters embedded in the Si nitride layers (Dal Negro et al., 2008). Figure 7 shows a TEM bright-field image of our sample in crosssection, taken using a JEOL 2010 TEM operated at $200 \mathrm{KV}$. The average amorphous Si (a-Si) and SRN layer thicknesses were measured to be $21 \mathrm{~nm}$ and $26 \mathrm{~nm}$, respectively. The structure of the sample consists of 10 SRN layers and 9 amorphous $\mathrm{Si}$ (a-Si) layers for a total thickness of $450 \mathrm{~nm}$. A higher magnification of a SRN layer shows the nucleation of amorphous Si nanocrystals marked by the arrows in the inset (panel b). The amorphous Si nanocrystals embedded in the Si nitride layers give rise to strong near-infrared photoluminescence with nanosecond decay dynamics at room temperature, as discussed in details elsewhere (Dal Negro et al., 2006; Dal Negro et al., 2008; Li et al., 2008).

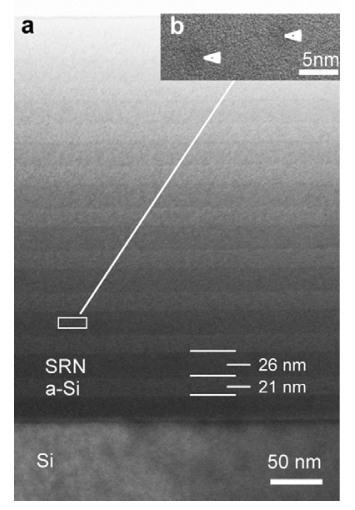

Fig. 7. (a) Cross sectional TEM bright-field micrograph of SRN/Si-SLs consisting of 10 SRN layers and 9 a-Si layers for a total thickness of $450 \mathrm{~nm}$. (b) Higher magnification of a SRN layer, showing amorphous Si nanocrystals marked by arrows.

Silicon nanocomposities sample was obtained by sol-gel technique. Si nanoparticles were obtained by crushing a silicon wafer and reducing their dimensions by a thermal dry oxidation. Afterward, an etching of the oxidized silicon nanoparticles was performed by a solution prepared by hydrofluoric acid and ethanol with a volumetric rate of $\mathrm{HF}: \mathrm{C}_{2} \mathrm{H}_{5} \mathrm{OH}=$ 1:1. The $\mathrm{SiO}_{2}$ sol was prepared by mixing the precursor tetraethyl orthosilicate $\mathrm{Si}\left(\mathrm{OC}_{2} \mathrm{H}_{5}\right)_{4}$ (TEOS-Sigma-Aldrich) with the solvent (94\% denaturated ethanol); an homogeneous and continuous film was obtained with a $0.5 \mathrm{M}$ solution. Acidulated water $(0.01 \mathrm{~N}$ $\mathrm{HCl}: \mathrm{CH}_{3} \mathrm{COOH}=1: 1$ ) was added in a hydrolysis ratio $\mathrm{HR}=4$. Fluorescéine was used as surfactant. Suspension was prepared mixing $5 \mathrm{ml}$ of $\mathrm{HF}: \mathrm{C}_{2} \mathrm{H}_{5} \mathrm{OH}=1: 1$ solution with silicon nanoparticles into $20 \mathrm{ml}$ of sol-gel solution. The biggest silicon particles were eliminated filtering the solution by a membrane with pore radius of $0.2 \mathrm{~mm}$. The deposition onto a glass substrate was realised by spin coating. Finally, two thermal treatment, for water and alcohol condensation, were performed. The thin-film so obtained has a thickness of $0.5 \mathrm{~mm}$ and its total length is $1.95 \mathrm{~cm}$. The sample was observed using a JEOL JEM 2010F STEM/TEM operated at $200 \mathrm{kV}$. In Fig.8(a) is shown the EFTEM image in cross view: in white contrast the shape of a crystalline silicon nanoparticle is shown. The STEM analysis in 
plan view configuration (Fig.8(b)) allowed us to evaluate the mean radius (49 $\mathrm{nm}$ ) of the silicon dots and also the dot density $\left(1.62 \times 10^{8}\right.$ dots $\left./ \mathrm{cm}^{2}\right)$ (Nicotra et al., 2004; Spinella et al., 2005; Nicotra et al., 2006).
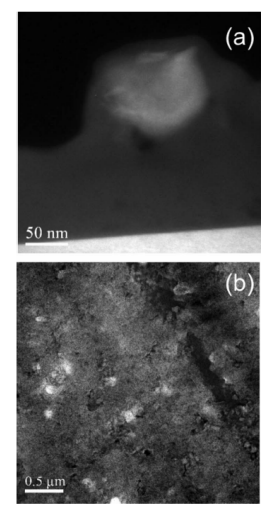

Fig. 8. (a) EFTEM micrograph taken at $16 \mathrm{eV}$. The white spot is a silicon nanoparticles embedded into the silica matrix. (b) STEM micrograph clearly detects Si nanoparticles, white spots, embedded into the silica matrix.

\subsection{SRS Measurements}

For both samples we carried out measures of SRS gain. In Fig. 9 and in Fig. 10 (Sirleto et al., 2008; Sirleto et al., 2009; Ferrara et al., 2010), the maxima of the signal wavelength scans are plotted as a function of the effective pump power (including the pass through the filter and objective) for both the SRN/Si-SLs sample and the sample of silicon nanocomposities, respectively.

For SRN/Si-SLs sample, the maximum signal gain obtained was $0.87 \mathrm{~dB} / \mathrm{cm}$, while in the case of the sample of silicon nanocomposities the maximum SRS gain obtained was 1.4 $\mathrm{dB} / \mathrm{cm}$. Both in Fig.9 and Fig.10, the SRS gain in a float zone high purity and high resistivity bulk silicon is also plotted as a function of the effective pump power. All plots in Fig.9 and in Fig.10 show an approximately linear dependence, as expected for the gain of a Raman amplifier as a function of pump power. As shown in Fig.9, SRN/Si-SLs exhibits a Raman gain significantly greater than bulk silicon. Although the estimation of the gain coefficient $g$ is not straightforward due to the uncertainty in the effective focal volume inside the sample, our data clearly demonstrate a value of $g$ which is about four time larger than the value reported for silicon (Ralston \& Chang, 1970) can be obtained in silicon nanostructures. Furthermore, our data prove a threshold power reduction of about $40 \%$ in silicon nanocrystals $\left(P_{\mathrm{th}} \approx 150 \mathrm{~mW}\right)$ with respect to silicon $\left(P_{\mathrm{th}} \approx 250 \mathrm{~mW}\right)$.

Raman amplifier based on silicon nanocomposites exhibits a SRS gain significantly greater than bulk silicon, as shown in Fig.10. By our data, a preliminary evaluation of approximately a fivefold enhancement of the gain coefficient in Raman amplifier based on silicon nanocomposities with respect to silicon is obtained (Ralston \& Chang, 1970). Furthermore our data prove a significant threshold power reduction (about 60\%) in silicon nanocomposities $\left(P_{\mathrm{th}} \approx 100 \mathrm{~mW}\right)$ with respect to silicon $\left(P_{\mathrm{th}} \approx 250 \mathrm{~mW}\right)$. 


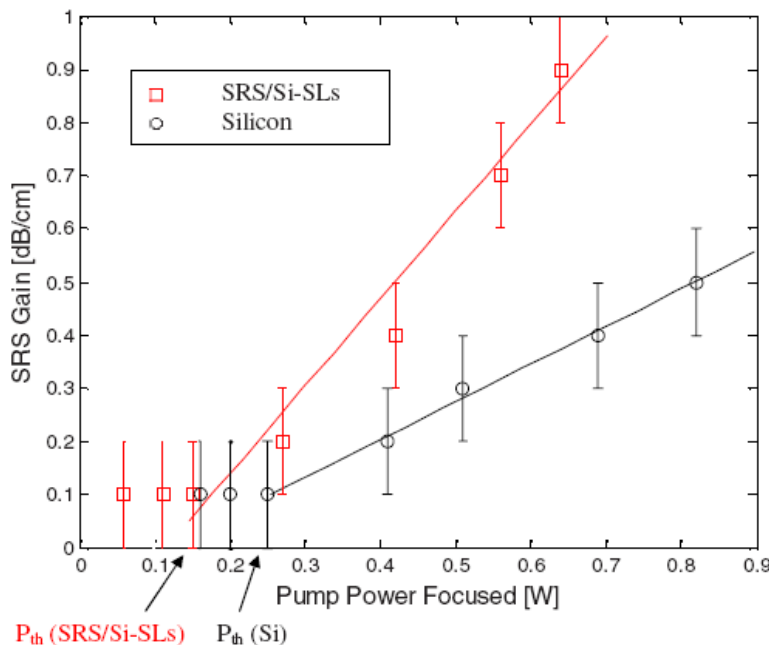

Fig. 9. The SRS-gain (amplification of the stokes signal in $\mathrm{dB} / \mathrm{cm}$ ) is plotted against the effective pump power at the sample surface both for amorphous Si nanoclusters embedded in SRN-Si-SLs ( $\square$ red) and for bulk silicon ( $\circ$ black).

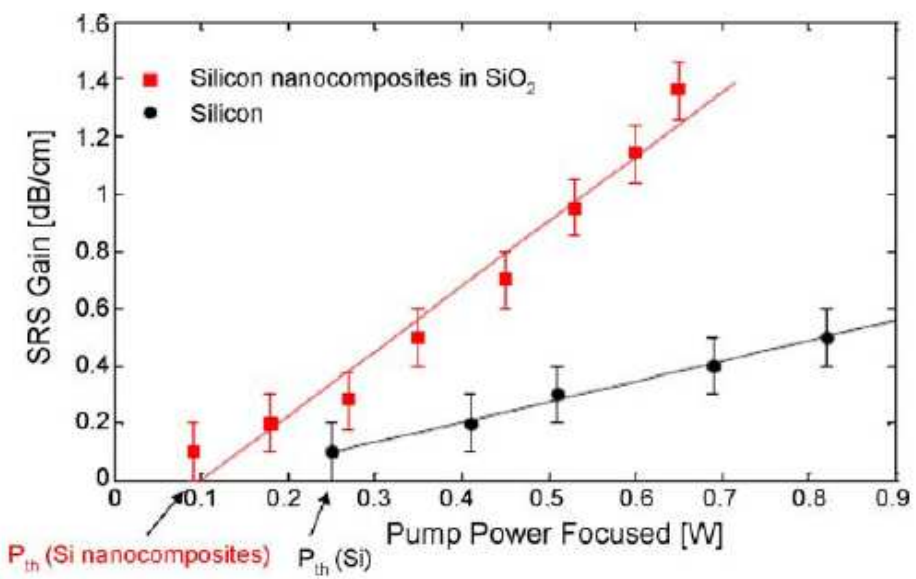

Fig. 10. The SRS gain (amplification of the stokes signal in $\mathrm{dB} / \mathrm{cm}$ ) is plotted against the effective pump power at the sample surface both for silicon nanocomposities in $\mathrm{SiO}_{2}$ ( $\square$ red) and for bulk silicon ( $\circ$ black).

\section{Discussion of results}

It is well known that third-order non-linear effects are generally characterized by the nonlinear absorption $(\beta)$ and the non-linear refractive index $(\gamma)$. The non-linear coefficients, namely $\beta$ and $\gamma$, are described by $a(I)=a_{0}+\beta I$ and $n(I)=n_{0}+\gamma I$ where $a_{0}$ and $n_{0}$ stand for the linear absorption and refractive index respectively. The $\beta$ and $\gamma$ values are used to evaluate the imaginary $\left(\operatorname{Im} \chi^{(3)}\right)$ and real $\left(\operatorname{Re} \chi^{(3)}\right)$ parts of the third-order non-linear susceptibility, 
respectively. We note that the real part describes the phenomena related to the intensity dependent index of refraction, while the imaginary part describes two photon absorption and SRS.

A way to enhance the real part of cubic nonlinearities in materials is to artificially 'shrinking' the electrons in regions much shorter than their natural delocalization length in the bulk. In such morphologies, optical resonances will usual appear, resulting from dielectric or quantum confinement, the former prevailing in metal nanocrystals, the latter prevailing in semiconductor nanocrystals. Quantum confinement occurs at a nanometer scale when the electron and hole envelope functions are restricted within a region whose spatial extension is lower than the exciton Bohr radius. This leads to quasi-discrete energy level structures, eventually showing sharp absorption lines. The concentration of the oscillator strength into these discrete levels leads to enhancement of the optical transitions rates and to a size-dependent nonlinear optical susceptibility (Klein, 1996).

Non resonant nonlinearities take place when the light linear absorption is negligible (at frequencies well below the absorption edge). They are related to the anharmonic motion (or virtual excitations) of bound electrons, and are very fast: typical recovery times are of the order of picoseconds. For $\chi^{(3)}$ based nonlinear devices, an enhancement of the real part of the third order nonlinear susceptibility in silicon nanostructure, due to quantum confinement, in the transparency range has been proved (Hernández et al., 2008). It is important to point out that the imaginary part of third order susceptibility of the nanostructured/nanocomposite materials, relating to the Raman gain coefficient, has been never theoretically investigated before. However, an enhancement of imaginary part of third order nonlinear susceptibility is expected.

In order to get Raman laser or amplifier in silicon, the main difficult was due to the presence of TPA, which reduces the efficiency of SRS. Taking advantage of Si-nc optical properties, we explore the possibility to reduce TPA and, at the same time, to enhance SRS.

It is well known that TPA process vanishes for $\hbar \omega<1 / 2 E_{g}, \hbar \omega$ being the photon energy and the band-gap. In Si-nc, due to quantum confinement, an increase of the band-gap with respect to the silicon is obtained. Reducing the dot (wires) dimensions, the band-gap increases. Therefore, considering a suitable size of the dot for which the relation is satisfied, the reduction of TPA is obtained (Lettieri \& Maddalena, 2002).

In recent years, there is a strong interest in investigation of Raman scattering in electronsconfined and photons-confined materials. The phenomenon of strong resonant and local enhancement of visible electromagnetic (EM) radiation when incident on the surface of metallic particles and films resulting from surface plasmon resonances continues to attract significant attention for fundamental and applied interests (Kawata et al., 2009). However, the possibility of enhancement of EM radiation from semiconducting and insulating materials, particularly in silicon, is noteworthy for silicon-based optoelectronic applications owing to the potential for monolithically integrating photonic technology and semiconductor electronics.

In the following discussion, we distinguish between Si-nc and silicon nanocomposities, because in the former (silicon nanocrystals) the mean radius of silicon dots was less than in the latter (low dimensional silicon). The different size of the particles corresponds to a fundamental difference between the two experiments reported in this chapter. Concerning 
stimulated Raman scattering in silicon nanostructures (SRN/Si-SLs), being the particle dimension of about $2 \mathrm{~nm}$, the phonon confinement effect is significant; therefore, we suggest that enhancement explanation has to be sought out in the framework of matter confinement and related to the enhancement of the third order nonlinear susceptibility in small Si clusters (approximately $2 \mathrm{~nm}$ in diameter) that nucleate in a high density inside silicon-rich nitride materials. However, the structure of the SRN/Si interfaces, the stoichiometric material disorder, and the cluster dimensionality are also important parameters that are expected to significantly influence Raman amplification, a theoretical understanding of their respective roles remains to be established.

Concerning stimulated Raman scattering in silicon nanocomposites in $\mathrm{SiO}_{2}$, being the diameter of particles dispersed in silica matrix of about 0.1 micron, the enhancement of SRS was due to a photons confinements effect. In order to try to explain why the presence of silicon particles can increase the Raman gain coefficient, we suggest two possible option. The former, it is well known that the nonlinear optical properties of composites material are characterized an "enhancement of local field" (Fischer et al., 1995). Off resonance, the electric field amplitude of an incident laser beam becomes no uniformly distributed between the two constituents of composite and the electric field strength within the more nonlinear constituent will exceed the spatially averaged field strength. Therefore, the effective real part of third order susceptibility of the composite can exceed that of each of its constituents (Fischer et al., 1995). At the same time, strong resonant Raman scattering in dielectric particles is obtained, when the wavelength of an incident field is commensurate with that of an electromagnetic eigenmode of the particle, which depends on its size and on the refractive indices of the particle and the surrounding medium (Murphy \& Brueck, 1983). This enhancement can be understood by viewing the particle as a cavity whose dimensions determine whether or not it is in resonance with either the incident or the emitted EM waves or both (i. e. double resonance). The observations of an enhancement of about 100 of the intensity of spontaneous Raman scattering were reported in Murphy \& Brueck (1983) from a variety of Si structures having submicrometer dimensions $(x \sim a / \lambda \sim 1)$, where $\lambda$ is the wavelength and $a$ is a characteristic particle dimension; for example spheres, having diameters of $\sim 0.1$ micron, were considered. As expected, the enhanced one-phonon spectra reported was a Lorentzian line shape with widths comparable with that of good-quality bulk $\mathrm{Si}$, while the exploited resonances were corresponding to electromagnetic cavity modes with at least one optical wavelength resonating within the particle.

The latter, is related to the optical transport properties of complex photonics structures on the intermediate regime between complete order or disorder. Light waves in disordered materials perform a random walk, which could lead to a multiple scattering process and to a strong localization of dielectric field (Wiersma et al., 2005). In a binary system with components of refractive indices $\mathrm{n} 1$ and $\mathrm{n} 2$, the efficiency of light scattering depends on how these components are organized in the system, the dimensions of the components, and the refractive index ratio $\mathrm{n} 1 / \mathrm{n} 2=\mathrm{m}$. In a specific regime, light propagation can be inhibited due to interference and the field intensity in localized regions can be significantly larger than in the surroundings (Schuurmans et al., 1999). As a consequences nonlinear optical properties of disordered material should be enhanced.

In our opinion the localization could play an important role on SRS and the combination of localization and SRS gain could be of particular interest for photonic application, where disordered materials could provide optical amplification via SRS. 


\section{Conclusions}

In this chapter, we experimentally investigate stimulated Raman scattering in amorphous $\mathrm{Si}$ nanocrystals and silicon nanocomposite and a number of advantages with respect to silicon were demonstrated.

First, according to phonon confinement model, two significant improvement of Raman approach in silicon quantum dots with respect to silicon were reported: the broadening of spontaneous Raman emission and the tuning of the Stokes shift. Considering silicon quantum dots having crystal size of $2 \mathrm{~nm}$, a significant broadening of about $65 \mathrm{~cm}^{-1}$ and a peak shift of about $19 \mathrm{~cm}^{-1}$ were obtained. Taking into account such results, more than the half of C-band telecommunication (width $=146 \mathrm{~cm}^{-1}$ ) could be covered using silicon quantum dots, without implementing the multi pump scheme.

Then, we experimentally demonstrate that amorphous $\mathrm{Si}$ nanocrystals and silicon nanocomposite can provide larger Raman gain values and a significant reduction in threshold power with respect to bulk Si devices.

We have two significant consequences related to our results. The first, concerning a fundamental point of view, the broadening of the Raman gain spectra combined with the present observation of enhanced Raman gain lead us to conclude that the traditional tradeoff between gain and bandwidth, valid in bulk materials, could be overcome in lowdimensional materials.

The second, concerning an applicative point of view, the possibility to enhance the Raman gain coefficient and to reduce two-photon absorption, at the same time, in silicon quantum dot was addressed. This means that the main limitation of first silicon Raman lasers could be overcame. Therefore, we quite optimistic believe that our experimental results can open the way to the fabrication of more efficient Raman lasers and amplifiers compatible with $\mathrm{Si}$ technology.

\section{Acknowledgment}

The authors would like to thank Luca Dal Negro, Soumendra N. Basu, Joe Warga and Rui Li for their contributions about the realization and the structural characterization of the sample of silicon nanocrystals embedded in Si-rich nitride/silicon superlattice structures. Moreover, we would like to thank Corrado Spinella and Giuseppe Nicotra for their help about the structural characterization of the sample of silicon nanocomposities embedded in $\mathrm{SiO}_{2}$.

\section{References}

Agrawal, G. P. (1995). Nonlinear Fiber Optics, 2nd ed. New York: Academic.

Boyd, R. W. (2003). Non linear optics, Second edition, 451-476, Academic Press, USA.

Boyraz, O. and Jalali, B. (2004). Demonstration of a silicon Raman laser, Opt. Express, Vol. 12, No. 21, pp. 5269-5273.

Campbell, H. and Fauchet, P. M. (1986). The effects of microcrystal size and shape on the one phonon Raman spectra of crystalline semiconductors, Solid State Commun., Vol. $58,739-741$. 
Cao, L.; Nabet, B.; Spanier, J. E. (2006). Enhanced Raman scattering from individual semiconductor nanocones and nanowires, Physical Review Letters, Vol. 96, no. 15, Article ID 157402, pp. 1-4.

Claps, R.; Dimitropoulos, D.; Han, Y. and Jalali, B. (2002), Observation of Raman emission in silicon waveguides at $1.54 \mu \mathrm{m}$, Opt. Express, Vol. 10, No. 22, pp. 1305-1313.

Claps, R.; Dimitropoulos, D.; Raghunathan, V.; Han, Y. and Jalali, B. (2003). Observation of stimulated Raman scattering in silicon waveguides, Opt. Express, Vol. 11, No. 15, pp. 1731-1739.

Claps, R.; Raghunathan, V.; Dimitropoulos, D. and Jalali, B. (2003). Anti-stokes Raman conversion in silicon waveguides, Opt. Express, Vol. 11, No. 22, pp. 2862-2872.

Claps, R.; Raghunathan, V.; Dimitropoulos, D. and Jalali, B. (2004). Influence of nonlinear absorption on Raman amplification in silicon waveguides, Opt. Express, Vol. 12, No. 12 , pp. $2774-2780$.

Dal Negro, L.; Yi, J. H.; Michel, J.; Kimerling, L. C.; Chang, T.-W. F.; Sukhovatkin, V.; Sargent, E. H. (2006). Light emission efficiency and dynamics in silicon-rich silicon nitride films, Appl. Phys. Lett., Vol. 88, pp. 233109.

Dal Negro, L.; Li, R.; Warga, J.; Basu, S. N. (2008). Sensitized Erbium emission from siliconrich nitride/silicon superlattice structures, Appl. Phys. Lett., Vol. 92, pp. 181105.

Dekker, R.; Usechak, N.; Först, M.; Driessen, A. (2007). Ultrafast nonlinear all-optical processes in silicon-on-insulator waveguides, J. Phys. D, Vol. 40, pp. R249-R271,

Dimitropoulos, D.; Jhaveri, R.; Claps, R.; S.Woo, J. C. and Jalali, B. (2005), Lifetime of photogenerated carriers in silicon-on-insulator rib waveguides, Appl. Phys. Lett., Vol. 86, No. 7, pp. 071115(1)-071115(3).

Dimitropoulos, D.; Fathpour, S.; Jalali, B. (2005). Intensity dependence of the carrier lifetime in silicon Raman lasers and amplifiers, Appl. Phys. Lett., Vol. 87, No. 26, pp. 261108(1)-261108(3).

Espinola, R. L.; Dadap, J. I.; Osgood, R. M. Jr.; McNab, S. J. and Vlasov, Y. (2004). Raman amplification in ultrasmall silicon-on-insulator wire waveguides, Opt. Express, Vol. 12, No. 16, pp. 3713-3718.

Fathpour, S.; Boyraz, O.; Dimitropoulos, D.; Jalali, B. (2006). Demonstration of CW Raman gain with zero electrical power dissipation in $\mathrm{p}$-i-n silicon waveguides, presented the IEEE Conf. Lasers and Electro-Optics (CLEO), Long Beach, CA, 2006, Paper CMK3.

Ferrara, M. A.; Donato, M. G.; Sirleto, L.; Messina, G.; Santangelo, S.; Rendina, I. (2008). Study of strain and wetting phenomena in porous silicon by Raman scattering, Journal of Raman Spectroscopy, Vol. 39, pp. 199-204.

Ferrara, M. A.; Sirleto, L.; Nicotra, G.; Spinella, C.; Rendina, I. (2010). Enhanced gain coefficient in Raman amplifier based on silicon nanocomposities, Photon Nanostruct: Fundam. Appl., http:/ / dx.doi.org/10.1016/j.photonics.2010.07.007

Freeouf, J. L.and Liu, S. T. (1995). Minority carrier lifetime results for SOI wafers, in Proc. IEEE Int. SOI Conf., Oct. 1995, pp. 74-75.

Fischer, G. L.; Boyd, R. W.; Gehr, R. J.; Jenekhe, S. A.; Osaheni, J. A.; Sipe, J. E.; WellerBrophy, L. A. (1995). Enhanced nonlinear optical response of composite materials, Phys. Rev. Lett., Vol. 74, pp. 1871.

Hart, T. R.; Aggarwal, R. L. and Lax, B. (1970). Temperature dependence of Raman scattering in silicon, Phys. Rev. B, Condens. Matter, Vol. 1, No. 2, pp. 638-642. 
Hernández; Pellegrino, P.; Martínez, A.; Lebour, Y.; Garrido, B.; Spano, R.; Cazzanelli, M.; Daldosso, N.; Pavesi, L.; Jordana, E.; Fedeli, J. M. (2008). Linear and nonlinear optical properties of $\mathrm{Si}$ nanocrystals in $\mathrm{SiO}_{2}$ deposited by plasma-enhanced chemical-vapor deposition, Journal of Applied Physics, Vol. 103, pp. 064309.

Islam, M.N. (2002). Raman amplifiers for telecommunications, IEEE J. Selected Top. Quantum Electron., Vol. 8, pp. 548-559.

Jalali, B.; Raghunathan, V.; Dimitropoulos, D.; Boyraz, O. (2006). Raman-based silicon photonics, IEEE J. Sel. Top. Quantum Electron., Vol. 12, pp. 412-421.

Jian Wu; Awnish K. Gupta; Humberto R. Gutierres, Eklund, P. C. (2009). Cavity-enhanced stimulated Raman scattering from short GaP nanowires, Nano Lett., Vol. 9, pp. 3252-3257.

Kawata, S.; Inouye, Y.; Verma, P. (2009). Plasmonics for near-field nano-imaging and superlensing, Nature Photonics, Vol. 3, no. 7, pp. 388-394.

Klein, L. C. (1996). Nanomaterials: Synthesis, Properties and Application, A. S. Edelstein and R. C. Cammarata, Institute of Physics Bristol-UK.

Lettieri, S. and Maddalena, P. (2002). Nonresonant Kerr effect in microporous silicon: Nonbulk dispersive behavior of below band gap $\chi_{(3)}(\omega)$, J. Appl. Phys., Vol. 91, 5564.

Li, R.; Schneck, J. R.; Warga, J.; Ziegler, L. D.; Dal Negro, L. (2008). Carrier dynamics and erbium sensitization in silicon-rich nitride nanocrystals, Appl. Phys. Lett., Vol. 93, pp. 091119.

Liang, T. K. and Tsang, H. K. (2004). Role of free carriers from two-photon absorption in Raman amplification in silicon-on-insulator waveguides, Appl. Phys. Lett., Vol. 84, No. 15, pp. 2745-2747.

Liu, A.; Rong, H.; Paniccia, M.; Cohen, O.; Hak, D. (2004). Net optical gain in a low loss silicon-on-insulator waveguide by stimulated Raman scattering, Opt. Express, Vol. 12, no. 18, pp. 4261-4267.

Mendicino, M. A. (1998). Comparison of properties of available SOI materials, in Properties of Crystalline Silicon, Hull, R.; Ed. London, U.K.: Inst. Eng. Technol., 1998 , ch. 18.1, pp. 992-1001.

Mori, A.; Masuda, H.; Shikano, K.; Shimizu, M. (2003). Ultra-wide-band tellurite-based fiber Raman amplifier, J. Lightwave Technol., Vol. 21, pp. 1300.

Murphy, D. V.; Brueck, S. R. J. (1983). Enhanced Raman scattering from silicon microstructures, Optics Letters, Vol. 8, pp. 494-496.

Nature News Editorial. First silicon laser pulses with life. Available: from http://www.nature.com/news/2004/041025/full/041025-10.html

Nicotra, G.; Puglisi, R.A.; Lombardo, S.; Spinella, C.; Vulpio, M.; Ammendola, G.; Bileci, M.; Gerardi, C. (2004). Nucleation kinetics of Si quantum dots on $\mathrm{SiO}_{2}$, J. Appl. Phys., Vol. 88, pp. 2049.

Nicotra, G.; Hui-Ting Chou, Henning Stahlberg, N.D. ; Browning, (2006). Avoiding charge induced drift in vitrified biological specimens through scanning transmission electron microscopy, Microsc. Microanal. Vol. 12, pp. 246-247.

OSA Press Room Editorial. Advancing the science of light. Available from: http://www.osa.org/news/pressroom/release/11.2002/jalali.aspx

Pavesi, L.; Lockwood, D.J. (2004). Silicon Photonics, Springer-Verlag, Berlin 2004. 
Ralston, J. M. and Chang, R. K. (1970). Spontaneous-Raman-scattering efficiency and stimulated scattering in silicon, Phys. Rev. B, Condens. Matter, Vol. 2, No. 6, pp. 1858-1862.

Ritcher, H.; Wang, Z. P.; Ley, L. (1981). The one phonon Raman spectrum in microcrystalline silicon, Solid State Commun., Vol. 39, pp. 625-629.

Rivero, C.; Richardson, K.; Stegeman, R.; Stegeman, G.; Cardinal, T.; Fargin, E.; Couzi, M.; Rodriguez, V. (2004). Quantifying Raman gain coefficients in tellurite glasses, $J$. Non-Cryst. Solids, Vol. 345-346, pp. 396-401,.

Rong, H.; Liu, A.; Jones, R.; Cohen, O.; Hak, D.; Nicolaescu, R.; Fang, A. and Paniccia, M. (2005). An all-silicon Raman laser, Nature, Vol. 433, No. 7023, pp. 292-294.

Rong, H.; Jones, R.; Liu, A.; Cohen, O.; Hak, D.; Fang, A. and Pannicia, M. (2005). A continuous-wave Raman silicon laser, Nature, Vol. 433, No. 7027, pp. 725-728.

Rong, H.; Kuo, Y. H.; Xu, S.; Liu, A.; Jones, R.; Paniccia, M. (2006). Monolithic integrated Raman silicon laser, Opt. Express, Vol. 14, No. 15, pp. 6705-6712.

Russell, J. P. (1965). Raman scattering in silicon, Appl. Phys. Lett., Vol. 6, No. 11, pp. 223-224.

Shen, Y.R.; Bloembergen, N. (1965). Theory of stimulated Brillouin and Raman scattering, Phys. Rev., Vol. 137, pp. A1787.

Schuurmans, F. J. P.; Vanmaekelbergh, D.; van de Lagemaat, J.; Lagendijk, A. (1999). Strongly Photonic Macroporous GaP Networks, Science, Vol. 284, pp. 141-143.

Sirleto, L.; Ferrara, M. A.; Rendina, I.; Jalali, B. (2006). Broadening and tuning of spontaneous Raman emission in porous silicon at 1.5 micron, Appl. Phys. Lett., Vol. 88, pp. 211105.

Sirleto, L.; Ferrara, M. A.; Rendina, I.; Basu, S.N.; Warga, J.; Li, R.; Dal Negro, L (2008). Enhanced stimulated Raman scattering in silicon nanocrystals embedded in siliconrich nitride/silicon superlattice structures, Applied Physics Letters, Vol. 93, pp. 251104.

Sirleto, L.; Ferrara, M.A.; Nicotra, G; Spinella, C; Rendina, I. (2009). Observation of stimulated Raman scattering in silicon nanocomposites, Appl. Phys. Lett., Vol. 94, pp. 221106.

Spillane, S. M.; Kippenberg, T. J.; Vahala, K. J. (2002). Ultralow-threshold Raman laser using a spherical dielectric microcavity, Nature, Vol. 415, pp. 621-623.

Spinella, C.; Bongiorno, C.; Nicotra, G.; Rimini, E.; Muscarà, A.; Coffa, S. (2005). Quantitative determination of the clustered silicon concentration in substoichiometric silicon oxide layer, Appl. Phys. Lett., Vol. 87, pp. 044102.

Soref, R. (2006). The Past, Present, and Future of Silicon Photonics, IEEE Journal of Selected Topics in Quantum Electronics, Vol. 12, No. 6, pp. 1678-1687.

Temple, P. A. and Hathaway, C. E. (1973). Multiphonon Raman spectrum of silicon, Phys. Rev. B, Condens. Matter, Vol. 7, No. 8, pp. 3685-3697.

Wiersma, D. S.; Sapienza, R.; Mujumdar, S.; Colocci, M.; Ghulinyan, M.; Pavesi, L. (2005). Optics of nanostructured dielectrics, J. Opt. A, Pure Appl. Opt., Vol. 7, pp. S190.

Zhang, B. P.; Shimazaki, K.; Shiokawa, T.; Suzuki, M.; Ishibashi, K.; Saito, R. (2006). Stimulated Raman scattering from individual single-wall carbon nanotubes, Appl. Phys. Lett., Vol. 88, pp. 241101. 


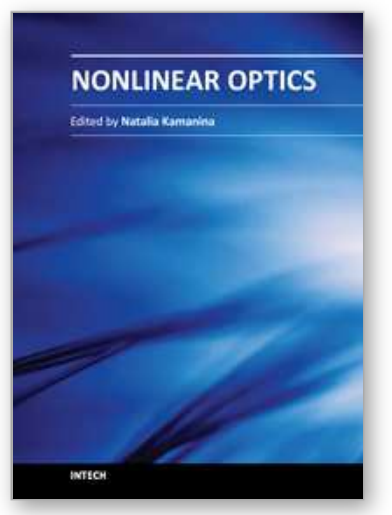

\author{
Nonlinear Optics \\ Edited by Dr. Natalia Kamanina
}

ISBN 978-953-51-0131-4

Hard cover, 224 pages

Publisher InTech

Published online 29, February, 2012

Published in print edition February, 2012

Rapid development of optoelectronic devices and laser techniques poses an important task of creating and studying, from one side, the structures capable of effectively converting, modulating, and recording optical data in a wide range of radiation energy densities and frequencies, from another side, the new schemes and approaches capable to activate and simulate the modern features. It is well known that nonlinear optical phenomena and nonlinear optical materials have the promising place to resolve these complicated technical tasks. The advanced idea, approach, and information described in this book will be fruitful for the readers to find a sustainable solution in a fundamental study and in the industry approach. The book can be useful for the students, post-graduate students, engineers, researchers and technical officers of optoelectronic universities and companies.

\title{
How to reference
}

In order to correctly reference this scholarly work, feel free to copy and paste the following:

M. A. Ferrara, I. Rendina and L. Sirleto (2012). Stimulated Raman Scattering in Quantum Dots and Nanocomposite Silicon Based Materials, Nonlinear Optics, Dr. Natalia Kamanina (Ed.), ISBN: 978-953-510131-4, InTech, Available from: http://www.intechopen.com/books/nonlinear-optics/stimulated-ramanscattering-in-quantum-dots-and-nanocomposite-silicon-based-materials

\section{INTECH}

open science | open minds

\author{
InTech Europe \\ University Campus STeP Ri \\ Slavka Krautzeka 83/A \\ 51000 Rijeka, Croatia \\ Phone: +385 (51) 770447 \\ Fax: +385 (51) 686166 \\ www.intechopen.com
}

\author{
InTech China \\ Unit 405, Office Block, Hotel Equatorial Shanghai \\ No.65, Yan An Road (West), Shanghai, 200040, China \\ 中国上海市延安西路65号上海国际贵都大饭店办公楼405单元 \\ Phone: +86-21-62489820 \\ Fax: +86-21-62489821
}


(C) 2012 The Author(s). Licensee IntechOpen. This is an open access article distributed under the terms of the Creative Commons Attribution 3.0 License, which permits unrestricted use, distribution, and reproduction in any medium, provided the original work is properly cited. 